\title{
LA EMPRESA SOCIAL. EXPERIENCIAS INNOVADORAS A TRAVÉS DEL DEPORTE
}

\author{
POR \\ Cristina LÓPEZ-CÓZAR NAVARRO ${ }^{1}$, \\ Tiziana PRIEDE BERGAMINI ${ }^{2}$ y \\ Javier DEL ARCO JUAN ${ }^{3}$
}

\section{RESUMEN}

Dentro del ámbito de la economía social destacan las empresas sociales, las cuales identifican y tratan de solucionar problemas sociales que las administraciones públicas no son capaces de solventar de la manera adecuada. Por su parte, la actual crisis económica ha aumentado las desigualdades sociales y ha provocado un aumento de la pobreza y del riesgo de exclusión social. En este nuevo escenario es donde el emprendimiento social está adquiriendo relevancia, pues es capaz de satisfacer las necesidades no atendidas, ni por las instituciones públicas, ni por el mercado. Respecto a las instituciones públicas, principalmente debido a la falta de recursos, y por lo que se refiere al mercado, por la falta de interés derivada de la escasa rentabilidad económica que presenta este tipo de actividades. La empresa social puede, en este contexto, rentabilizar su actuación social innovadora al dar protagonismo por encima de todo a las personas en lugar del capital. En este sentido, su finalidad social y la reinversión de sus beneficios económicos en su propio objetivo, propician que este tipo de entidades sean idóneas para poder beneficiar a las comunidades en las que están asentadas. Dentro de los ámbitos donde el emprendimiento social puede tener una mayor incidencia se encuentra el deporte. De hecho, a lo largo del presente estudio se pretende poner de manifiesto la importancia y la idoneidad del deporte como ámbito para el desarrollo del emprendimiento social, ya que ambos buscan fines semejantes: la igualdad de oportunidades, la cohesión y la integración entre otros, junto con el logro de una sociedad más equitativa, dentro de un proyecto social responsable en todas sus dimensiones.

\footnotetext{
${ }^{1}$ Universidad Politécnica de Madrid. Dirección de correo electrónico: cristina.lopezcozar@upm.es

${ }^{2}$ Universidad Europea de Madrid. Dirección de correo electrónico: tiziana.priede@uem.es

${ }^{3}$ Universidad Europea de Madrid. Dirección de correo electrónico: javier.arco@uem.es

REVESCO No 119 - Tercer Cuatrimestre 2015 - ISSN: 1885-8031 - www.ucm.es/info/revesco

http://dx.doi.org/10.5209/rev_REVE.2015.n119.49069
}

Fecha de recepción: 23/12/2014

Fecha de aceptación: 24/03/2015 
Palabras clave: valor social, desarrollo, economía social, instituciones deportivas, integración.

Claves Econlit: L830, L200, L260, L300.

\begin{abstract}
Within the field of social economy, social enterprises stand out as organizations which identify and try to solve social problems that public administrations are not able to. Meanwhile, the current economic crisis has increased social inequalities and has led to increased poverty and risk of social exclusion. In this new scenario social entrepreneurship is gaining relevance, because it is able to meet social needs unattended by public institutions or by the market; due mainly to lack of resources in the case of public institutions, but also due to the lack of sufficient profitability, in the case of the market. The social enterprise can, however, make profitable such innovative social actions, and give prominence to people rather than capital. In this sense, the social purpose and the reinvesting of profits is its own goal, and fosters such entities to benefit the communities which they belong to. One of the areas where social entrepreneurship can have a high impact is in sport. In fact, the main objective of this study is to highlight the importance and appropriateness of sport as an area for development of social entrepreneurship. Both seek similar ends, which include equal opportunities, cohesion and integration, along with achieving a more equitable and responsible society.
\end{abstract}

Keywords: Social economy, integration, development, sport organizations.

\title{
1. INTRODUCCIÓN
}

La empresa social es aquella que identifica un problema social o ambiental y pretende solucionarlo a partir de una idea innovadora. Uno de los objetivos prioritarios de este tipo de empresas es la ayuda a personas que se encuentran en una situación delicada, derivada de problemas económicos, físicos, o de cualquier otra naturaleza. Con este propósito de luchar contra la exclusión social, y siendo capaz de llegar allí donde las instituciones públicas no pueden hacerlo, el ámbito deportivo se presenta como una posibilidad para lograr esta inserción (Demoustier, 2005).

La actual situación de crisis económica ha provocado un importante desarrollo de las empresas sociales en multitud de países de todo el mundo (Short, Moss y Lumpkin, 2009; 
Santos, 2012). Paralelamente, también en la comunidad científica y académica ha incrementado el interés sobre esta realidad, por lo que se ha aumentado considerablemente la investigación en este campo (Zahra, Gedajlovic, Neubaum y Shulman, 2009). Así, en la última década se pueden encontrar numerosos trabajos teóricos y empíricos enfocados a su estudio; sin embargo, para el caso particular de la empresa social en el deporte, los trabajos son mucho más escasos. Ante esta situación, creemos que hay un importante vacío en la literatura y que se trata de una línea de investigación interesante que está aún por desarrollar. Por este motivo, este artículo tiene como principal objetivo contribuir a mejorar el conocimiento sobre la empresa social, profundizar en la importante función que cumple en la sociedad, y presentar diferentes ejemplos y experiencias de su implantación en el ámbito deportivo.

En definitiva, el presente trabajo pretende poner de manifiesto mediante un estudio descriptivo, que el ámbito deportivo ofrece una excelente oportunidad para la puesta en marcha de iniciativas empresariales innovadoras encaminadas a la consecución de un mundo más equitativo y responsable, esto es, y en línea con Zhara et al. (2009) y Shepherd y Patzelt (2011), mejorar la riqueza social de las personas y de la comunidad en general. Para conseguir este objetivo, tras la introducción, se realiza una aproximación a la economía social y al concepto de empresa social; en el siguiente epígrafe, se muestran diferentes modalidades del deporte y su vinculación con objetivos sociales a partir de diferentes vertientes tales como la salud, la integración o la educación; a continuación, se presentan varias experiencias y prácticas de emprendimiento social en el deporte. Finalmente, se exponen las principales conclusiones del trabajo.

\section{LA EMPRESA SOCIAL}

En Europa, el origen del concepto de empresa social se sitúa a principios de los años 90 del siglo pasado (Galera y Borzaga, 2009; Defourny y Nyssens, 2012). Su nacimiento es paralelo al desarrollo de la economía social, en la que se enmarcan a una amplia variedad de organizaciones de diversas formas jurídicas, creadas con el objetivo de dar respuesta a las necesidades compartidas por determinados colectivos sociales (Monzón, 2006; Priede, LópezCózar y Rodríguez, 2014).

La Comisión Europea ofrece diferentes vías para el desarrollo de este tipo de organizaciones y otorga gran importancia a este sector, por lo que ha propiciado la puesta en 
marcha de numerosas iniciativas y proyectos de investigación. Entre ellos destaca el desarrollado por la Red Europea de Investigación (Emergence del Entreprises Sociales en Europe, EMES), que surge con el propósito de crear un marco común que permita identificar a las empresas sociales en Europa, y al mismo tiempo, que sea suficientemente amplio como para dar cabida a las diferencias propias de cada país (Borzaga y Defourny, 2001; Galera y Borzaga, 2009; Fisac-García, Alves de Carvalho, Moreno-Romero, Moreno-Mateos y Rojas, 1012).

Pese a que son muchas las aproximaciones realizadas por diferentes autores al concepto de empresa social (Zahra et al. 2009; Moreira, Urriolagoitia y Vernis, 2011), EMES propone un modelo basado en una serie de indicadores, que ha supuesto una de las identificaciones de empresa social que mayor aceptación ha tenido. Se trata de nueve indicadores que tradicionalmente se han presentado en dos grupos, cuatro criterios económicos y cinco sociales (Borzaga y Defourny, 2001; Defourny y Nyssens, 2008); si bien recientemente, Defourny y Nyssens (2012) plantean presentar dichos indicadores en tres subgrupos, añadiendo la dimensión gobierno participativo a las dos anteriores; de esta forma, señalan los autores, es más sencillo reconocer ciertas particularidades que caracterizan a muchas de las organizaciones propias de la economía social.

En cualquier caso, los nueve criterios que permiten identificar a las empresas sociales, con independencia de la forma legal que estas adopten, son los siguientes: a) las empresas sociales se dedican a la producción de bienes o prestación de servicios, y tienen que mantener un contacto permanente con el mercado, pues deben competir con las empresas tradiciones; $b$ ) los fundadores deben asumir un cierto nivel de riesgo económico puesto que la empresa tiene que ser auto sostenible a partir de la actividad que se realiza, esto es, no son organizaciones caritativas ni pueden depender de donaciones o subvenciones; c) tienen que poseer fuerza de trabajo remunerada; d) han de tener un claro objetivo de beneficio para la comunidad; e) debe implicar a un grupo de ciudadanos de forma colectiva para cubrir una necesidad; f) debe poseer una distribución de beneficios limitada para evitar la maximización de los mismos; g) han de tener autonomía en su administración, es decir, no es parte de ninguna otra organización, sino que tienen que tener sus propios estatutos y desarrollarlos de manera independiente; h) el poder de decisión no debe basarse en la propiedad del capital; finalmente, i) es preciso que tengan una gestión participativa que involucre a diversas partes interesadas en el negocio. 
A partir de estos indicadores, la Comisión Europea considera que una empresa social es aquella que actúa en el mercado, ofreciendo a la sociedad bienes y servicios de manera empresarial, pero que se caracteriza por tres elementos fundamentales: realiza su actividad de manera socialmente innovadora, al ser el objetivo social su principal razón de ser; sus beneficios se reinvierten principalmente en la realización de dicho objetivo social; y tienen un modo de organización y un régimen de propiedad también condicionados por esta misión social, basados en principios tales como democracia, responsabilidad, participación o justicia social (COM, 2011; Enciso, Gómez y Mugarra, 2012).

De esta manera, la Comisión no pretende dar una definición normativa, sino, más bien, una descripción de las características comunes a las empresas sociales existentes en la mayoría de los Estados miembros, con el propósito de respetar las diferentes opciones económicas, políticas y sociales posibles en Europa, y sólo adoptará una definición más precisa en caso de que sea necesario por temas reglamentarios (Priede et al., 2014). Partiendo de esta proposición, capaz de abarcar las peculiaridades propias de los distintos países de la Unión Europea, cada uno de ellos perfilará el enfoque que mejor se adapte a sus necesidades. Por lo tanto, según la concepción que se tenga del Estado del Bienestar, del modelo de prestación de servicios (público, privado o mixto), y de los papeles asumidos por el Estado y la sociedad civil, cada país redefine el concepto de empresa social (Defourny y Nyssens, 2008; Fisac-García et al., 2012).

En el caso de España, la economía social y las entidades que la integran tienen su primer reconocimiento legal en 1990, en la Ley 31/1990, de 27 de diciembre, de Presupuestos Generales del Estado, si bien su presencia en la realidad económica de nuestro país es anterior (Fajardo, 2012a). Actualmente, la delimitación de este sector viene marcada por la Ley 5/2011, de 29 de marzo, de Economía Social, donde se define como el conjunto de actividades económicas y empresariales que llevan a cabo aquellas entidades de ámbito privado, que persiguen el interés general económico o social, o ambos.

La propia Ley establece una serie de principios que rigen la economía social, que giran en torno a la primacía de las personas y del fin social sobre el capital, tanto en la gestión como en la toma de decisiones; la aplicación de los resultados obtenidos en función del trabajo realizado por sus miembros, y en su caso, al objeto social de la entidad; el compromiso con el desarrollo local, la igualdad de oportunidades entre hombres y mujeres, la cohesión social, la inserción de personas en riesgo de exclusión social, la generación de empleo estable y de 
calidad, la conciliación y la sostenibilidad; y, finalmente, la independencia manifiesta con respecto a los poderes públicos. Sin embargo, se decide adoptar el criterio de que ser una entidad lucrativa o no serlo, no sea una condición determinante ni excluyente para formar parte de la economía social, si bien sí se señala la importancia de la no maximización de los beneficios económicos (Crespo, 2013).

Siguiendo la tradición histórica europea, la Ley 5/2011 opta por asumir una concepción amplia de la economía social, incluyendo a las cooperativas, las sociedades laborales, las mutualidades, los centros especiales de empleo, las empresas de inserción, las cofradías de pescadores, las sociedades agrarias de transformación, las asociaciones y las fundaciones que lleven a cabo actividad económica, así como cualquier otra entidad que cumpla los principios antes mencionados. Se establece así un marco jurídico común para todas las entidades que constituyen la economía social, pero respetando la normativa específica vigente aplicable a cada una de ellas, al tiempo que deja abierta la posibilidad de incorporar nuevas entidades a la economía social (Paniagua, 2011; Fajardo, 2012b).

Finalmente, la Ley propone una serie de políticas de apoyo a la economía social, definiendo medidas como la de aminorar los obstáculos en la creación y desarrollo de estas entidades, eliminando trabas y simplificando los trámites administrativos; promocionar la formación y la readaptación profesional en este ámbito; facilitar el acceso a las innovaciones, etc. En definitiva, crear un entorno favorable para el estímulo de la economía social y la promoción de las entidades que la integran. No obstante, si bien la Ley despertó grandes expectativas, la realidad es que muchos de los objetivos propuestos están aún pendientes de aplicación (Chaves y Savall, 2013).

Una vez delimitado el concepto de economía social, se puede decir que una empresa social es aquella que, independientemente de la forma jurídica que adopte, persigue un objetivo social por encima de la búsqueda de riqueza de sus propietarios. Se trata de empresas que no son ni públicas ni capitalistas tradicionales y que tienen una clara vocación de servicio a la comunidad. Operan en el mercado, a través de la producción de bienes o la prestación de servicios, y ofrecen soluciones innovadoras, eficaces y eficientes, a necesidades sociales y medio ambientales aún no resueltas. 


\section{EL DEPORTE COMO SECTOR IDÓNEO PARA EL DESARROLLO DEL EMPRENDIMIENTO SOCIAL}

Definir el deporte, como han señalado diversos autores (Cagigal, 1985; Gratton y Taylor, 2000; Olivera, 2006) es una tarea realmente compleja. En una primera aproximación, según el Diccionario de la Real Academia Española, se entiende por deporte la "actividad física, ejercida como juego o competición, cuya práctica supone entrenamiento y sujeción a normas"

Rodgers (1977) considera que en el deporte deben estar presentes idealmente cuatro elementos, y señala que debe implicar "actividad física", ser practicado con un "objetivo recreativo", implicar un "elemento de competición" y desarrollarse en el marco de una “organización institucional”. Cagigal (1985) propone una de las definiciones más completas de deporte, considerándolo como cualquier tipo de actividad física que se realiza con el deseo de compararse o superar, tanto a otros como a uno mismo, o realizada en general con intención lúdica o gratificadora, a pesar del esfuerzo; así mismo, este autor señala que puede incluir desde un gran espectáculo, hasta una competición de nivel modesto. Más adelante, el propio Consejo de Europa en la Carta Europea de Deportes, aprobada en 1992, (Consejo de Europa, 1992) utiliza una definición muy concisa, señalando en su artículo 2: "se entenderá por deporte todo tipo de actividades físicas que, mediante una participación, organizada o de otro tipo, tengan por finalidad la expresión o la mejora de la condición física y psíquica, el desarrollo de las relaciones sociales o el logro de resultados en competiciones de todos los niveles".

De las anteriores definiciones se deduce que el término deporte es un concepto abierto que se refiere a una amplia variedad de actividades, que pueden ser practicadas con diferentes objetivos (competición, ocio, salud, etc.) y que abarca múltiples aspectos de diferentes ámbitos (educativo, social, económico, etc.). Así, siguiendo a Olivera (2006), para un mejor estudio y comprensión del concepto, se pueden identificar una serie de factores endógenos (que corresponden a su lógica interna), entre los que se incluye el juego, la competición, el esfuerzo físico, las reglas, el espíritu deportivo y el juego limpio (fairplay), y la institucionalización. Estos factores, los cuales aparecen en diferente proporción en función de los intereses que los motiven, constituyen el núcleo común del deporte. Por su parte, desde su

\footnotetext{
${ }^{4}$ En una segunda acepción: recreación, pasatiempo, placer, diversión o ejercicio físico, por lo común al aire libre.
} 
lógica externa, es importante señalar que el deporte interactúa con su entorno, de manera que influye y a su vez es influido por el contexto social, cultural, político, económico, tecnológico y medioambiental. Por lo tanto, si bien resulta difícil formular una definición concreta de deporte, sí es posible identificarlo a partir de la correcta congruencia entre los factores endógenos y exógenos citados.

Por otra parte, el deporte puede ser clasificado siguiendo diferentes criterios (modalidad, complejidad, hábito, finalidad, etc.). Si se atiende a los aspectos sociológicos, se puede establecer la siguiente clasificación: deporte organizado formalmente en clubes y federaciones, deporte de grupos espontáneos, deporte institucional (practicado en el seno de una institución) y deporte comunicativo o espectáculo (emitido por medios de comunicación).

El proyecto europeo COMPASS (Coordinated Monitoring of Participation in Sports) propone una clasificación del deporte en función del grado de hábito por parte del participante (TNS Opinion and Social, 2010), distinguiendo entre práctica intensa de modo competitivo u organizado, práctica intensa, práctica regular de modo competitivo y organizado, práctica regular de modo recreativo, práctica irregular, práctica ocasional y práctica nula.

Hernández y Gallardo (1994) realizan una clasificación del deporte basándose en los ámbitos y la intención del mismo, distinguiendo entre el deporte en la escuela, obligatorio, con carácter formativo y con intención educativa; el deporte como ocio, actividad física, libre, voluntaria y espontánea, dirigida a toda la población y con intención lúdica; y el deporte de competición, no espontáneo, con el objetivo del perfeccionamiento y con la intención de búsqueda de máximo rendimiento.

Por su parte, en la Carta Europea del Deporte para Todos (Consejo de Europa, 1980), se señala la influencia social del deporte y su importancia en el desarrollo de la salud física y metal de las personas, y se ofrece una clasificación de las actividades dividiendo el deporte en cuatro grandes categorías:

- Juegos y deportes de competición, en los cuales, los participantes aceptan unas reglas y se enfrentan a otros.

- Actividades de aire libre, caracterizadas por tratar de dominar un determinado terreno (montaña, agua, bosque, etc.) venciendo las dificultades del terreno escogido y otros factores como las condiciones meteorológicas. 
- Actividades estéticas, en las que el individuo dirige su atención sobre sí mismo y realiza un movimiento corporal coordinado (por ejemplo el baile, el patinaje artístico o ciertas formas de gimnasia rítmica).

- Actividades de mantenimiento de la condición, es decir, ejercicios que persiguen una mejoría o mantenimiento de la forma física y una sensación de bienestar general.

No obstante, la mayoría de las clasificaciones del deporte generalmente aceptadas atienden a la finalidad o la función del mismo. De esta manera, se pueden destacar fundamentalmente dos modalidades básicas (Cagigal, 1975): deporte como espectáculo o deporte competición, y deporte como práctica apta para todos o deporte-ocio.

En la misma línea, del Arco y Rodríguez (2013a) proponen una clasificación similar a la anterior, estableciendo dos categorías:

- Deporte amateur o aficionado. En este sentido, tal y como establece la Carta Olímpica, la práctica deportiva puede considerarse un derecho humano, de manera que cualquier persona tenga la posibilidad de practicar deporte, sin ningún tipo de discriminación y dentro del espíritu olímpico, que exige comprensión mutua, solidaridad, espíritu de amistad y juego limpio (fairplay). Se puede decir que esta categoría estaría compuesta por el deporte utilitario, el deporte educativo y el deporte recreativo.

- Deporte profesional en las diferentes competiciones y las diferentes modalidades deportivas. Es lo que se puede denominar deporte espectáculo o de competición.

Todas estas clasificaciones muestran que el deporte, en sus diferentes variantes, modalidades y productos, constituye una magnífica oportunidad para la puesta en marcha de proyectos empresariales dentro del ámbito del emprendimiento social, es decir, dirigidos a lograr una sociedad más equitativa dentro de un proyecto responsable.

No obstante, y para que quede más patente ese vínculo entre deporte y emprendimiento social, se debe considerar la conexión que tiene el deporte y la sociedad a través de tres vertientes fundamentales: la salud, la integración social y la educación. Efectivamente, el deporte constituye un elemento primordial en la sociedad moderna y en el sistema educativo de todos los países. La práctica de cualquier modalidad deportiva es fundamental para el mantenimiento de la salud y, por tanto, tal y como establece el Preámbulo 
de la Ley 10/1990, de 15 de octubre, del Deporte, "es un factor corrector de desequilibrios sociales que contribuye al desarrollo de la igualdad entre los ciudadanos, crea hábitos favorecedores de la inserción social $\mathrm{y}$, asimismo, su práctica en equipo fomenta la solidaridad". Todas estas características configuran la práctica deportiva como ejercicio físico, en sus distintas modalidades, como un elemento determinante de la calidad de vida y la utilización activa y participativa del tiempo de ocio en la sociedad contemporánea. De hecho, el deporte se considera una vía adecuada para mejorar la salud de las personas en sus diferentes etapas de vida, desde la infancia hasta la madurez, adaptándolo a las características y los requerimientos propios de cada edad. Así mismo, se puede considerar una valiosa herramienta a través de la cual se pueden transmitir valores y competencias, tales como responsabilidad, trabajo en equipo, solidaridad, igualdad, etc., fomentando con ello una educación integral. Finalmente, respecto a la inclusión social, el deporte también ayuda a la integración de todas aquellas personas que por diversos motivos se encuentren aislados o fuera del sistema.

Por su parte, su práctica es fundamental en el desarrollo de la sociedad y de los individuos que la integran, lo cual se manifiesta en las dos modalidades anteriormente establecidas: deporte profesional y deporte amateur o aficionado. En cuanto al deporte profesional, este se ha convertido en una actividad atractiva y rentable, tanto para los deportistas, como para los directivos, los propietarios, los inversores, los intermediarios, los agentes y los patrocinadores; en este sentido, el deporte persigue como objetivo fundamental ofrecer un espectáculo, normalmente de masas. Respecto a la actividad deportiva no profesional o de aficionado, tiene como objetivo simplemente la libre práctica del deporte, y pretende llegar al mayor número de aficionados posible. Esta es la variante en la que el emprendimiento social presenta mayores posibilidades de desarrollo.

La práctica deportiva como aficionado es básica en la sociedad moderna en las tres líneas argumentales comentadas: la salud, la integración social y la educación. Diversas investigaciones realizadas sobre la relación entre el deporte y la salud, han demostrado los efectos beneficiosos de la práctica continuada de actividades deportivas (World Health Organization, 2003; del Arco y Rodríguez, 2013b). Al mismo tiempo, el deporte es un elemento de cohesión y relación social, que ayuda al desarrollo de la personalidad y al establecimiento de relaciones entre sus practicantes, con el resultado positivo que implica. Por tanto, además de los efectos sobre la salud, el deporte supone educación -el deporte inculca 
valores- e integración social (del Arco y Rodríguez, 2013b), tal y como se pone de manifiesto en el desarrollo que el Consejo Superior de Deportes realiza en 2011 con la publicación Deporte, actividad física e inclusión social: Una guía para la intervención social a través de las actividades deportivas (Carranza y Maza, 2011).

Es preciso considerar, por un lado, el importante aumento en el número de deportistas profesionales en todo el mundo, y en particular en España; y, por otro lado, el aumento del tiempo libre y de ocio en la sociedad empleado, en parte, en la práctica deportiva. Así, por ejemplo, en España, alrededor de 16 millones de personas mayores de 14 años practican deporte (García Ferrando y Llopis, 2011). Estos dos aspectos muestran la importancia del deporte en las dos modalidades señaladas. Hoy en día, esta relevancia de la actividad deportiva es reconocida en la mayoría de los países desarrollados. De hecho, los poderes públicos de nuestro país en los principios rectores de la política social y económica abordan esta dimensión del deporte. En concreto, en el capítulo tercero del título I de la Constitución, en su artículo 43.3, se señala que "los poderes públicos fomentarán la educación sanitaria, la educación física y el deporte. Asimismo, facilitarán la adecuada utilización del ocio". De dicho artículo se puede extraer la actitud que han de tener los poderes públicos ante las dos modalidades deportivas ya señaladas (deporte profesional y aficionado). Dicha actitud ha de ser de fomento y decidido apoyo al deporte-práctica y de prudente distanciamiento del deporte profesional.

De esta forma, basándose en la propia Constitución española, los poderes públicos tienen entre sus competencias y deberes la obligación de estimular y promocionar la práctica deportiva, ya sea mediante el ejercicio de sus funciones o mediante el reconocimiento y estímulo de las funciones desarrolladas por el sector privado en la mencionada promoción deportiva, ajustándose a "los principios de colaboración responsable entre todos los interesados" (Art. 1 de la Ley 10/1990, de 15 de octubre, del Deporte). Al hacer referencia a que los poderes públicos, deben fomentar la práctica deportiva, se hace referencia tanto al Estado (Administración Central del mismo) ${ }^{5}$, como a las Entidades Locales (Ayuntamientos) ${ }^{6}$

\footnotetext{
${ }^{5}$ Ver preámbulo de la Ley del Deporte y el art. 149 de la Constitución española de 1.979. Se justifica la intervención del Estado en el fomento del deporte a tenor de la legislación y de los títulos competenciales en los que se desarrolla el deporte: educación, investigación, sanidad o legislación mercantil; competencias que avalan la actuación estatal en el deporte a nivel supraautonómico o estatal.

${ }^{6}$ V. art. 25.2, m y 26.1,c de la Ley Básica de Régimen Local. Estas Entidades tienen competencias en materia deportiva, sobre todo, en aquellos municipios que superen los 20.000 habitantes.
} 
y a las Comunidades Autónomas ${ }^{7}$; dándose incluso actuaciones coordinadas y de cooperación entre la Administración del Estado y la de las Comunidades Autónomas para aquellas competencias concurrentes que sin duda propician una política deportiva más dinámica y con efectos multiplicadores, tal y como menciona el Preámbulo de la Ley 10/1990, de 15 de octubre, del Deporte.

Como ha quedado de manifiesto, los poderes públicos están encargados de la promoción del deporte. No obstante, debido a diversos factores, entre ellos la actual crisis económica en los diferentes países occidentales y, en concreto en España, ha provocado que los presupuestos de las diferentes administraciones se vean reducidos (como consecuencia de las políticas de reducción de déficit público). Las partidas que más impacto están teniendo son las relacionadas con desarrollo e integración social y las deportivas.

Debido a que el deporte en sus múltiples modalidades se ha visto mermado en su financiación pública, deja abierta la vía para que la iniciativa privada, a través del emprendimiento social, se haga cargo de las competencias que la mano visible del Estado no es capaz de atender. Además, como se ha puesto de relieve, el deporte es una industria o sector que permite alcanzar los objetivos que persigue el emprendimiento social, centrándose en la búsqueda de la equidad y del desarrollo social a través de diferentes proyectos innovadores basados en la actividad deportiva.

\section{DESCRIPCIÓN DE EXPERIENCIAS INNOVADORAS EN EL DEPORTE}

Una vez planteadas las bases teóricas sobre las que se asienta la empresa social descritas en el primer apartado del presente artículo y justificada la idoneidad del sector del deporte para el desarrollo de los emprendedores sociales, en este epígrafe se exponen algunas experiencias llevadas a cabo en los últimos años, tanto en España como en otros países. Dado que el deporte permite el desarrollo de prácticas activas y participativas, se configura como un factor corrector de desequilibrios sociales, fomenta la solidaridad y la igualdad, y favorece la inserción social. Así, en los ejemplos detallados se puede observar cómo efectivamente, a través de iniciativas de emprendimiento social basadas en actividades deportivas, se resuelven diferentes problemas tales como la inclusión de personas con discapacidad, el crecimiento y la mejora de las condiciones laborales en países en vías de desarrollo o la integración de colectivos en riesgo de exclusión social.

\footnotetext{
${ }^{7}$ El punto 19 del artículo 148.1 de la Constitución de 1.978 señala la "promoción del deporte y la adecuada utilización del ocio" como una de las competencias que han de asumir las Comunidades Autónomas.
} 
Este estudio, realizado mediante la descripción de casos, busca identificar patrones y proporcionar información que no podría obtenerse utilizando técnicas cuantitativas más restrictivas, por lo que es una metodología que resulta adecuada para la investigación en el área de las ciencias sociales (Alvord et al., 2004; Yin, 2008). Para ello, se pueden utilizar diversas fuentes como documentos, entrevistas, registros de archivos, observación directa, etc. (Chetty, 1996; Martínez, 2006). En el presente artículo se ha utilizado la información disponible en sus respectivas páginas de internet, y en algún caso, la información se ha ampliado con entrevistas personales realizadas a los emprendedores sociales que las han constituido.

A continuación se presentan cinco experiencias de emprendimiento social en el deporte. En primer lugar, se describen tres iniciativas que se han desarrollado en España; posteriormente se presenta otra con sede en Nairobi, aunque con cierto impulso británico; para finalizar con otro proyecto de origen norteamericano, pero con el sistema de fabricación localizado en Pakistán.

En el año 2000 nace en Madrid la fundación También (deporte adaptado para la inclusión de personas con discapacidad) como institución de interés general, cultural, deportivo y sin ánimo de lucro. Es creada por la emprendedora social Teresa Silva, deportista y empresaria, quien tras sufrir un grave accidente de parapente en el año 1989, mientras se entrenaba para los campeonatos del mundo, queda discapacitada en una silla de ruedas, lo cual le hizo afrontar las dificultades que este tipo de personas encuentran a la hora de desarrollar sus vidas. El objetivo de esta empresa social es pues la integración de personas con discapacidad psíquica, física y/o sensorial, mediante el fomento de actividades deportivas adaptadas, de ocio y de tiempo libre.

Esta empresa social se desarrolla principalmente a través de tres áreas de trabajo: en primer lugar, la organización de actividades, cursos y otros eventos, así como proporcionando el material adaptado para facilitar el acceso a estas actividades. En segundo lugar, la formación, asesoramiento y asistencia técnica a las personas con discapacidad, a sus familias y a personal especializado y otros voluntarios, para poder desarrollar con éxito el deporte adaptado. Finalmente, la realización de una importante labor de difusión a través de la organización de congresos, charlas, foros, exhibiciones y competiciones. 
En nuestro país, el desarrollo del deporte adaptado es relativamente reciente y su puesta en práctica requiere de un importante esfuerzo personal y económico, ya que los equipamientos son muy costosos y hacen falta muchas personas especializadas para apoyar su desarrollo, como fisioterapeutas, monitores especializados, voluntarios, etc. Así, gracias a la labor de esta empresa social que combina deporte e inclusión social, las personas con discapacidad pueden integrarse con normalidad en la sociedad junto a familiares y amigos, desarrollar la autoestima y disfrutar como cualquier otra persona del contacto con la naturaleza. En definitiva, incrementar su bienestar y su confianza, así como obtener una gran satisfacción personal.

Se trata de un primer caso que ilustra la relación entre la empresa social y la actividad física, presentando una alternativa para la integración de personas con discapacidad, basada en la experiencia personal de su fundadora, si bien, al tratarse de una fundación no cumple con todos los criterios establecidos por la definición de empresa social que ofrece el enfoque EMES. No obstante, como ya se apuntó, la Comisión no pretendía imponer una definición normativa, sino una descripción general de las características comunes a las empresas sociales. Por su parte, la Ley 5/2011 incluye a las fundaciones con actividad económica dentro del concepto de Economía Social, por lo que consideramos que a pesar de su sencillez, se trata de un ejemplo interesante de emprendimiento social en el deporte.

Inspirasports es una empresa social española que nace en enero del año 2010 como asociación sin ánimo de lucro con el objetivo de dar soporte al gobierno e instituciones públicas y privadas para elaborar iniciativas de desarrollo social a través de actividades deportivas. Los emprendedores sociales que llevaron a cabo esta iniciativa son Pedro Díaz Ridao y Germán Argüelles, quienes decidieron aunar sus esfuerzos tras su experiencia profesional en el ámbito de la gestión deportiva de la competición de alto nivel, experimentando de forma personal el inmenso potencial del deporte como herramienta para el desarrollo de personas en riesgo de exclusión social. Esta empresa se desarrolla principalmente a través de dos áreas de trabajo: la sensibilización e integración social, llevada a cabo a través de diversas actividades y proyectos deportivos, y la educación en valores y formación de personas en el uso de esta potente herramienta para que se conviertan también en agentes de cambio.

Respecto al área de sensibilización e integración social, destaca el proyecto Inspirarunning. Se trata de un club en el que participan voluntarios junto a personas en riesgo 
de exclusión social (como personas sin hogar, desempleados o inmigrantes con escasos recursos), cuyo objetivo es ayudar a este colectivo a mejorar su confianza y autoestima, así como su independencia y autosuficiencia, fomentando unas rutinas positivas y saludables a través de la carrera (runing). Este proyecto no persigue dotar a los afectados con medios materiales, sino por el contrario, pretende proporcionarles el apoyo emocional que les permita paulatinamente salir de su situación apoyándose en su propio esfuerzo. En efecto, la carrera basada en la igualdad y el respeto mutuo, cubre determinadas necesidades básicas, como la pertenencia al grupo, la motivación, la valoración de los logros y el sentirse querido y apreciado. Por su parte, esta actividad física también produce sensación de bienestar y ayuda a mejorar el funcionamiento intelectual. Además, si es reforzado por una persona cercana, labor que es realizada por los voluntarios y las personas que participan en el proyecto, provoca un impacto aún mayor en la autoestima. La labor de la empresa social en este proyecto consiste, por tanto, en buscar medios económicos y materiales para poder proporcionar a estas personas en riego de exclusión social material deportivo y un plan de entrenamiento y seguimiento, que les permita preparar carreras populares en las que poder medir sus logros.

En cuanto al área de formación y educación en valores, destacan el proyecto Sports Ethical Seal (SES) y el proyecto y la formación en el deporte para el desarrollo. SES es un sello de garantía ético que permite ayudar a las entidades deportivas (clubes, escuelas, federaciones, etc.) a desarrollar procedimientos y rutinas que permitan promover un comportamiento ético en el desarrollo de sus actividades deportivas. El sello ofrece un reconocimiento público del compromiso de la entidad deportiva en el fomento de los valores éticos y entornos deportivos estables que permitan a los niños ${ }^{8}$ desarrollar su actividad de manera positiva, saludable y en armonía con un entorno igualitario, solidario y responsable. Finalmente, en cuanto a la formación en el deporte, destacan sus esfuerzos por ampliar contenidos en materia de deporte para el desarrollo en el ámbito universitario a través de su difusión en diversos programas de grado y postgrado, con el fin de difundir entre los jóvenes universitarios el poder del deporte como herramienta para llevar a cabo el cambio social.

Se trata de una iniciativa con un nivel de complejidad más alto que la anterior, en el que los emprendedores sociales implicados desarrollan su idea de negocio con un claro enfoque de innovación social, explotando el gran potencial que presenta la carrera como

\footnotetext{
${ }^{8}$ El fomento de valores y comportamientos éticos desde edades muy tempranas es fundamental. Se considera idóneo en los períodos de educación infantil y primaria, para que puedan posteriormente ser desarrollados en la adolescencia y la juventud.
} 
herramienta para el desarrollo de personas en riesgo de exclusión social. Quizás este segundo caso se acerca más al concepto desarrollado por la Comisión, si bien, también se configura como una asociación sin ánimo de lucro dentro del concepto Economía Social.

Finalmente en cuanto a las iniciativas que han tenido lugar en nuestro país, cabe mencionar la reciente puesta en marcha del proyecto Sport2help (el deporte para ayudar), plataforma de micro aportaciones o crowdfunding, que pretende ayudar a desarrollar proyectos solidarios a través del deporte.

El crowdfunding es un nuevo método para la financiación de una amplia variedad de iniciativas -empresas, actividades culturales, proyectos sociales, etc.- que permite a los emprendedores sociales llevar a cabo su idea mediante la solicitud de fondos a un elevado número de personas (Belleflamme, Lambert y Schwienbacher, 2014; Mollick, 2014). Es una oferta abierta, planteada normalmente a través de internet, para la provisión de recursos, que pueden ser recibidos con o sin contraprestación (Kleemann, Vob y Rieder, 2008; Schwienbacher y Larralde, 2010). Así, en un proceso de crowdfunding intervienen tres grupos de participantes diferentes: el emprendedor, el grupo formado por los individuos que deciden aportar fondos para apoyar la iniciativa y las plataformas que permiten el contacto entre ambos (Quero y Ventura, 2014).

La plataforma Sport2help desarrolla el proceso de crowdfunding pero de forma original, ya que pone en contacto a un deportista que se marca un reto concreto (challenge) con el fin de recaudar fondos para una causa social defendida por una organización no gubernamental o entidad no lucrativa. Por su parte, cualquier persona que esté interesada en colaborar puede acceder a la plataforma y participar de forma directa en la financiación de dichas causas (helper). Lo interesante del proyecto es que canaliza el deporte y los retos marcados por los propios deportistas, como vía a través de la cual realizar los donativos, los cuales pueden hacerse desde 2 euros. En este caso, el crowdfunding es del tipo denominado de donación, es decir, la participación de las personas que deciden aportar fondos está motivada por el deseo de acceder al resultado esperado del proyecto, sin esperar ninguna contraprestación económica, como en los proyectos solidarios o humanitarios.

Entre los retos marcados por los deportistas se pueden citar la carrera Transvulcania de 73,3 Km de distancia; la realización en equipo de una larga marcha de 100km en un máximo de 32 horas denominado Trailwalker; otras pruebas de resistencia como triatlones y 
otras carreras de larga duración, etc. Se trata de marcar un reto difícil de lograr, que suponga un esfuerzo considerable para el deportista implicado en alcanzarlo. Los deportistas, por su parte, se vinculan a proyectos solidarios como: La Casa dels Xuklis, una ONG que gestiona una casa de acogida para las familias que tienen que desplazarse lejos de sus hogares para recibir tratamiento oncológico; Oxfam Intermón, a través del desarrollo proyectos que facilitan el acceso al agua potable a aquéllos más desfavorecidos; la Fundación Ared, dedicada a lograr la inserción social y laboral de mujeres en riesgo de exclusión social; o las risas de los Pallapupas, dedicados a aliviar el dolor de los niños que están en los hospitales aquejados de enfermedades graves. Así, cuando se accede a la plataforma, se puede elegir al deportista, su reto y la organización a la que dirige los fondos recaudados.

En definitiva, Sport2help se encarga de poner en contacto a los deportistas, las organizaciones sin ánimo de lucro y el público en general, con fin de mejorar el bienestar social, utilizando el deporte como instrumento para la creación de valor social. Se trata pues de una forma alternativa de innovación social basada en el crowfunding que emplea el deporte como medio para conseguir sus objetivos, pero no de un ejemplo concreto de emprendimiento social como el que se describe en los demás casos propuestos; no obstante, nos parece interesante su inclusión ya que este tipo de financiación participativa está experimentando un profundo desarrollo en los últimos años, y se configura como un mecanismo de financiación adecuado a las características de los emprendedores sociales (Lehner, 2013).

En cuanto a las iniciativas internacionales de emprendimiento social en el deporte, en primer lugar se describe Alive and Kicking, creada por Jim Cogan en el año 2004 en Nairobi, e impulsada por el departamento británico de desarrollo internacional Departament for International Development (DFID) ${ }^{9}$. Desde su nacimiento se estableció que la empresa debía utilizar los excedentes procedentes de la fabricación y venta de balones deportivos de diverso tipo para la solución de determinados problemas sociales en zonas desfavorecidas del continente africano. Así, la empresa crea empleo digno en varias comunidades de Kenya, Zambia and Ghana, incrementando la salud y la calidad de vida de los jóvenes en riesgo de exclusión, y ayuda a los niños de estas regiones a ejercer su derecho al juego. A lo largo de los últimos diez años, la empresa ha incrementado su fabricación, produciendo unos 60.000 balones al año y empleando alrededor de 120 personas.

\footnotetext{
${ }^{9}$ Institución pública del gobierno británico cuyo principal objetivo es trabajar para erradicar la pobreza extrema en el mundo a través de la financiación de organizaciones que se enfocan en esta dirección, como es el caso de Alive and Kicking. Este departamento desarrolla directamente, en veintiocho países de África, Asia y el Oriente Medio, varias líneas de trabajo tales como, educación, salud y crecimiento económico.
} 
Uno de los aspectos más relevantes llevados a cabo por esta organización es la concienciación social a través del deporte acerca de la transmisión de enfermedades contagiosas, dado el elevado número de habitantes de estos lugares que las padecen. En efecto, la empresa considera que el deporte es una herramienta excelente para la transmisión de mensajes saludables a los jóvenes. De esta manera, organiza torneos de fútbol y otros eventos combinando el juego y la educación sobre salud, con el fin de llamar la atención e incrementar el conocimiento de los jóvenes acerca de estas enfermedades, así como la realización gratuita de pruebas de diagnóstico para reducir su propagación.

En este caso se presenta una alternativa más en línea con el modelo de empresa social que se está consolidando en los últimos años en Europa. Reino Unido es uno de los países más avanzados en cuanto al desarrollo de un marco jurídico adaptado a este tipo de iniciativas (Priede et al., 2014) y a través del DFID, financia a este tipo de emprendedores. Así, resulta muy acertado aplicar una actividad económica rentable (fabricación y venta de balones de fútbol) para la solución de determinados problemas sociales en zonas desfavorecidas del continente africano y al mismo tiempo, utiliza el deporte como medio para transmitir mensajes saludables entre los jóvenes africanos para la mejora de hábitos saludables y la prevención de enfermedades.

En la misma línea que el ejemplo anterior, creada en el año 2010 en Estados Unidos, Senda -comercio justo en balones de futbol- es otro ejemplo de la aplicación del emprendimiento social en el deporte. En efecto, Santiago Halty, se plantea la industria del futbol desde una perspectiva diferente y la analiza con espíritu crítico, llegando a la conclusión de que las condiciones laborales y la calidad de las materias primas en la fabricación de balones de fútbol no cumplían, en la mayoría de los casos, con los mínimos exigidos, mientras por otro lado se empleaban grandes sumas de dinero en campañas publicitarias y promociones comerciales. Halty descubre que la pasión por este deporte de masas podía ser canalizada hacia la mejora de la calidad de vida de muchas personas. Así pues, Senda nace con el compromiso de fabricar y distribuir balones (y otro equipamiento) de forma responsable y sostenible a través del comercio justo. Este comercio se basa en el diálogo, la trasparencia y el respeto, garantizando los derechos de los productores y trabajadores generalmente establecidos en zonas marginadas (Salvá y Doblas, 2005).

La actividad de Senda se centra en tres aspectos fundamentales. Por una parte en los consumidores, es decir, en todos aquellos que practican el deporte para el que necesitan 
emplear productos de calidad con los que poder alcanzar su máxima capacidad. Por otra parte, en sus trabajadores; la empresa ha establecido su fábrica en Sialkot (Pakistan), la cual está certificada como comercio justo, lo que implica que los empleados desarrollan su actividad en condiciones adecuadas, recibiendo un salario justo y no permitiendo la mano de obra infantil. Finalmente, la empresa ha adquirido un compromiso con determinadas organizaciones sin ánimo de lucro tales como Street soccer, Bopr y Soccer withoutborders, las cuales emplean el futbol como medio para conseguir la integración de personas en riesgo de exclusión, con discapacidad, o jóvenes marginados. Así, Senda patrocina sus actividades y administra material deportivo para que estas organizaciones puedan alcanzar sus objetivos sociales.

Este emprendedor social ha identificado una interesante oportunidad de negocio basada en el deporte, cuyo principal objetivo es la resolución de varios problemas sociales ya que, no sólo contribuye al desarrollo y la mejora de las condiciones laborales en Pakistán, sino que con los rendimientos obtenidos por la fabricación y venta de balones, apoya a otras organizaciones que también están trabajando en la resolución de dificultades sociales, esta vez generadas en Estados Unidos. Por lo tanto, el modelo de negocio responsable que plantea esta iniciativa cumple con las bases del emprendimiento social citadas al principio de este trabajo ya que, sobre la base de un negocio rentable, está consiguiendo la creación de valor social y es en esta dirección en la que deberían plantearse las iniciativas de este tipo.

\section{CONCLUSIONES}

El actual escenario internacional, agravado por la situación de crisis económica, financiera y de valores, está promoviendo que en los últimos años se haya incrementado notablemente el interés por las empresas sociales y otras formas alternativas de asignación de recursos. Concretamente, la empresa social nace por la iniciativa y el empuje de una persona con un fuerte espíritu emprendedor y gran conciencia social, que pretende resolver un problema, normalmente reservado al Estado o a la iniciativa pública, empleando para ello modelos de negocio existentes en la economía de mercado, y generando con ello un impacto favorable en la comunidad y un importante valor social.

Al igual que las demás empresas, las de iniciativa social deben ser rentables y tener un resultado positivo que les permita financiar su actividad y asegurar su subsistencia; sin embargo, a diferencia de las sociedades mercantiles, el éxito de una empresa social no se mide en términos de resultados financieros obtenidos, sino por el efecto cualitativo y cuantitativo 
que tiene su actuación en beneficio de los colectivos a los que se dirige. En definitiva, además de cumplir con las exigencias que impone el orden económico de mercado, las empresas sociales deben, también, demostrar la creación de valor social.

En Europa se concede gran importancia a la empresa social, desde su origen a finales de los años noventa y durante su desarrollo enmarcado dentro de la denominada economía social, la cual por su parte, recoge diversos tipos de organizaciones con formas jurídicas diferentes, orientadas a sectores de actividad muy variados, precisamente creadas con el fin de responder a las necesidades sociales de determinados colectivos. Por su parte, en España, la economía social tiene también su origen en los años noventa, y actualmente se encuentra regulada por la Ley 5/2011, de 29 de marzo de economía social, en la que se sientan las bases de su funcionamiento. Es pues en este contexto en el que se desarrolla la empresa social en nuestro país, donde también puede adoptar diversas formas jurídicas y ejercer su actividad económica y social en cualquier sector de actividad. Existe pues un marco jurídico común a todas las entidades que constituyen la economía social, pero dejándose abierta la posibilidad de incorporar nuevas entidades, en línea con los principios de apertura y amplia concepción marcados por la normativa europea.

En el ámbito deportivo, el emprendimiento social encuentra un terreno lleno de oportunidades utilizando el deporte como herramienta para fomentar soluciones a determinados problemas sociales, tales como la reinserción de personas en riesgo de exclusión social, la inclusión personas con discapacidad o la creación de actividades para diversos colectivos (niños, adolescentes, parados, ancianos, etc.), entre otros. En efecto, la comunidad deportiva puede actuar de manera innovadora con el fin de crear un beneficio social y económico para la sociedad en su conjunto, al tiempo que realiza una actividad económica sostenible, capaz de crear riqueza, empleo y valor social.

La principal aportación del presente trabajo es precisamente poner de manifiesto la existencia de una gran oportunidad para poder desarrollar el emprendimiento social a través del deporte, tal y como se ha puesto de manifiesto en los diversos casos analizados, así como animar a los jóvenes de nuestro país a emprender una actividad de estas características con amplias expectativas de futuro y crecimiento. Esto tiene implicaciones tanto para los futuros emprendedores, como para las administraciones públicas. Respecto a los primeros, en momentos delicados como los que atraviesa nuestro país, y cuando se percibe una cierta recuperación económica, el emprender una actividad que permita el autoempleo, así como la 
posibilidad de crear otros puestos de trabajo y, al mismo tiempo, consiga la creación de valor social sostenible en el tiempo, se percibe como una posibilidad profesional muy recomendable.

Por su parte, respecto a las administraciones públicas, estas encuentran en el emprendimiento social interesantes soluciones a los problemas que las afectan. Así, la creación y desarrollo de estas iniciativas empresariales deben ser impulsadas y favorecidas a través de una serie de medidas concretas como el desarrollo de marcos legales y fiscales específicos para estos tipos especiales de empresas; darles prioridad en el acceso a contratos con el gobierno; incrementar las alternativas y facilidades a la hora de acceder a las fuentes de financiación; eliminar obstáculos para su creación; promover el uso de las innovaciones disponibles; impulsar programas de formación para los futuros emprendedores y abrir nuevas líneas de investigación para mejorar su conocimiento. De hecho, consideramos que debe fomentarse su difusión, tanto en la educación primaria y secundaria, como en la universidad, ya que el emprendimiento social es aún desconocido entre la mayoría de los jóvenes españoles como alternativa profesional, y generalmente confundido con las organizaciones no gubernamentales o la filantropía al tratarse de causas similares.

Si bien la Ley 5/2011 propuso políticas de apoyo para la creación y desarrollo de estas entidades y generó muy buenas expectativas, no obstante, la realidad en estos últimos años ha demostrado que los objetivos propuestos no se han cumplido y queda todavía mucho trabajo por desarrollar en este ámbito. Por su parte, en la Ley 14/2013, de 27 de septiembre, de apoyo a los emprendedores y su internacionalización, destaca en su exposición de motivos la alta tasa de desempleo juvenil que afecta a nuestra sociedad. En particular, señala que para el caso de los menores de 25 años se duplica la media europea, por lo que insiste en la necesidad de mejorar la eficacia de las políticas de apoyo institucional al emprendimiento, a través de iniciativas públicas de información, asistencia, asesoramiento y fomento de la cultura emprendedora, así como la concesión de ayudas o financiación. Sin embargo, en la citada Ley no se hace alusión al emprendimiento social, ni se fomentan medidas específicas adaptadas a este tipo de emprendimiento.

En definitiva, con este trabajo hemos querido poner de manifiesto la excelente oportunidad que ofrece el deporte para el fomento y desarrollo de iniciativas de emprendimiento social y la importancia de este tipo de actividades para nuestra sociedad en el momento actual. Consideramos que se trata de una buena coyuntura para la creación de un 
entorno favorable para su desarrollo, similar al que existe en otros países de nuestro entorno como es el caso del Reino Unido, que permita estimular la economía social y las diversas organizaciones que la componen.

\section{BIBLIOGRAFÍA}

ALVORD, S. H., BROWN, L. D. y LETTS, C. W. (2004) Social entrepreneurship and societal transformation an exploratory study. The Journal of Applied Behavioral Science, vol. $40, \mathrm{n}^{\mathrm{o}} 3$, pp. 260-282.

BELLEFLAMME, P. LAMBERT, T. y SCHWIENBACHER, A. (2014) Crowdfunding: Tapping the right crowd. Journal of Business Venturing, vol. 29, nº 5, pp. 585-609.

BORZAGA, C. y DEFOURNY, J. (Eds.) (2001) The Emergence of Social Enterprise. London y New York: Routledge.

CAGIGAL, J. M. (1975) El Deporte en la Sociedad Actual. Madrid: Prensa Española.

CAGigAL, J. M. (1985) Pedagogía del deporte como educación. Revista de Educación Física. Renovación de teoría y práctica, $\mathrm{n}^{\circ} 3$, pp. 5-11.

CARRANZA, M. y MAZA, G. (Coords.) (2011) Deporte, actividad física e inclusión social. Una guía para la intervención social a través de las actividades deportivas. Madrid: Consejo Superior de Deportes.

CHAVES, R. y SAVALL, T. (2013) La insuficiencia de las actuales políticas de fomento de cooperativas y sociedades laborales frente a la crisis en España. REVESCO. Revista de Estudios Cooperativos, $\mathrm{n}^{\circ} 113$, pp. 61-91.

CHETTY S. (1996) The case study method for research in small- and médium - sized firms. International small business journal, vol. 15, $\mathrm{n}^{\mathrm{o}} 1$, pp. 73-85.

CRESPO, T. (2013) Una nueva relación del Tercer sector y la economía social. Cuadernos de Trabajo Social, vol. $26 \mathrm{n}^{\circ}$ 1, pp. 65-74.

CONSEJO DE EUROPA (1980) European Sport for All Charter, Estrasburgo: Consejo de Europa.

CONSEJO DE EUROPA (1992) European Sports Charter, Estrasburgo: Consejo de Europa.

DEFOURNY, J. y NYSSENS, M. (2008). Social enterprise in Europe: Recent trends and developments. Social Enterprise Journal, vol 4 nº 3, pp. 202-228.

DEFOURNY, J. y NYSSENS, M. (2012) El enfoque EMES de la empresa social desde una perspectiva comparada. CIRIEC-España, revista de economía pública, social y cooperativa, $\mathrm{n}^{\circ} 75$, pp. 7-34. 
DEL ARCO, J. y RODRÍGUEZ, A. (2013a) Los Participantes en la Industria del Deporte, Revista Internacional de Derecho y Gestión del Deporte, n 24, pp. 22-32.

DEL ARCO, J. y RODRÍGUEZ, A. (2013b) Interrelación entre Actividad Deportiva y Desarrollo Socio-económico, Revista Internacional de Deportes Colectivos, nº 16, pp. 12 21.

DEMOUSTIER, D. (2005) Las empresas sociales: ¿nuevas formas de Economía Social en la creación de servicios y empleos? CIRIEC-España, revista de economía pública, social y cooperativa, $\mathrm{n}^{\circ}$ 52, pp. 219-236.

ENCISO, M., GÓMEZ, L. y MUGARRA, A. (2012) La iniciativa comunitaria en favor del emprendimiento social y su vinculación con la economía social: una aproximación a su delimitación social. CIRIEC-España, revista de economía pública, social y cooperativa, $\mathrm{n}^{\circ}$ 75 , pp. $55-80$.

FAJARDO, G. (2012a) El fomento de la “economía social” en la legislación española. REVESCO. Revista de Estudios Cooperativos, $\mathrm{n}^{\circ}$ 107, pp. 58-97.

FAJARDO, G. (2012b). El concepto legal de economía social y la empresa social. Revista Vasca de Economía Social-Gizarte Ekonomia Euskal Aldizkaria, no 8, pp. 63-84.

FISAC-GARCÍA, R., ALVES DE CARVALHO, N., MORENO-ROMERO, A., MORENOMATEOS, J. y ROJAS, A. (2012) Iniciativas emprendedoras de lucha contra problemas sociales: distintas aproximaciones geográficas. En: XVI Congreso de Ingeniería de Organización, 1065-1072, Vigo.

GALERA, G. y BORZAGA, C. (2009) Social enterprise: an international overview of its conceptual evolution and legal implementation. Social Enterprise Journal, vol. 5, nº 3, pp. 210-228.

GARCÍA FERRANDO, M. y LLOPIS, R. (2011) Ideal Democrático y Bienestar Personal. Encuesta sobre los hábitos deportivos en España 2010. Madrid: Consejo Superior de Deportes.

GRATTON, C. y TAYLOR, P. (2000) Economics of Sport and Recreation. Londres: Routledge.

HEINEMANN, K. (1998) Introducción a la economía del deporte. Barcelona: Paidotribo.

HERNÁNDEZ, M. y GALLARDO, L. (1994) Marco conceptual: las actividades deportivorecreativas. Apunts de Educación Física y Deportes, nº 37, pp. 58-67.

KLEEMANN, F., VOB, G. y RIEDER, K. (2008) Un (der) paid Innovators: The Commercial Utilization of Consumer Work through Crowdsourcing. Science, Technology \& Innovation Studies, $\mathrm{n}^{\circ} 4$, pp. 5-26. 
LEHNER, O. (2013) Crowdfunding social ventures: a model and research agenda. Venture Capital, vol 15, n 4, pp. 289-311.

MARTÍNEZ, P. C. (2006) El método de estudio de caso: Estrategia metodológica de la investigación científica. Pensamiento y gestión: Revista de la división de Ciencias Administrativas de la Universidad del Norte, vol. 20, pp. 165-193.

MOLLICK, E. (2014) The dynamics of crowdfunding: An exploratory study. Journal of Business Venturing, vol. 29, $\mathrm{n}^{\circ} 1$, pp. 1-16.

MONZÓN, J. L. (2006) Economía Social y conceptos afines: fronteras borrosas y ambigüedades conceptuales del Tercer Sector. CIRIEC-España, revista de economía pública, social y cooperativa, $\mathrm{n}^{\circ} 56, \mathrm{pp} .9-24$.

MOREIRA, P; URRIOLAGOITIA, L. y VERNIS, A. (2011) El emprendimiento social. Revista Española del Tercer Sector, $\mathrm{n}^{\mathrm{o}}$ 17, pp. 17-40.

OLIVERA, J. (2006). Hacia una nueva comprensión del deporte. Factores endógenos y exógenos. Apunts: Educación Física y Deportes, nº 86, pp. 3-6.

PANIAGUA ZURERA, M. (2011) Las empresas de la economía social más allá del comentario a la Ley 5/2011, de economía social. Madrid: Marcial Pons.

PRIEDE, T., LÓPEZ-CÓZAR, C. y RODRÍGUEZ, Á. (2014) Análisis del marco económicojurídico específico para los emprendedores sociales. Un estudio comparado entre diversos países. CIRIEC-España, revista de economía pública, social y cooperativa, $\mathrm{n}^{\circ}$ 80, pp. 428.

QUERO M.J. y VENTURA, R. (2014) Co-creación de valor. Un estudio de casos de crowdfunding. Universia Business Review, n 3, pp. 128-143.

SALVÁ, A. y DOBLAS, N. (2005) El comercio justo: implicaciones económicas y solidarias. CIRIEC-España, revista de economía pública, social y cooperativa, $\mathrm{n}^{\circ}$ 51, pp. 7-24.

SANTOS, F. M. (2012) A positive theory of social entrepreneurship. Journal of Business Ethics, vol. 111, nº 3, p. 335-351.

SCHWIENBACHER, A. y LARRALDE, B. (2010) Crowdfunding of small entrepreneurial ventures. SSRN Electronic Journal, Working Paper.Handbook of entrepreneurial finance, Oxford University Press, Forthcoming.

SHEPHERD, D. A., y PATZELT, H. (2011) The new field of sustainable entrepreneurship: studying entrepreneurial action linking "what is to be sustained" with "what is to be developed". Entrepreneurship Theory and Practice, vol. 35 nº1, pp. 137-163. 
SHORT, J. C., MOSS, T. W. y LUMPKIN, G. T. (2009) Research in social entrepreneurship: Past contributions and future opportunities. Strategic Entrepreneurship Journal, vol. 3, $\mathrm{n}^{\circ}$ 2, pp. 161-194.

TNS OPINION AND SOCIAL (2010) The Citizens of the European Union and Sport. Special Eurobarometer 334, Sport and Physical Activity. Bruselas: Comisión Europea.

WORLD HEALTH ORGANIZATION (2003) Health and development through physical activity and sport. Ginebra: World Health Organization Health Education and Health Promotion Unit.

YIN, R. K. (2008) Case study research: Design and methods (applied social research methods). Sage Publications, London.

ZAHRA, S. A., GEDAJlOVIC, E., NEUBAUM, D. O. y SHULMAN, J. M. (2009) A typology of social entrepreneurs: Motives, search processes and ethical challenges. Journal of Business Venturing, vol. 24, nº 5, pp. 519-532.

\section{PÁGINAS DE INTERNET CONSULTADAS}

ALIVE AND KICKING http://www.aliveandkicking.org/ (web en línea) (Consulta 9-122014).

DEPARTAMENT FOR INTERNATIONAL DEVELOPMENT (web en línea) https://www.gov.uk/government/organisations/department-for-international-development (consultado 5-12-2014).

INSPIRASPORT (web en línea) http://www.inspirasports.org/wordpress/ (consultado 5-122014).

SPORTS2HELP (web en línea) http://sport2help.org/ (consultado 6-12-2014).

FUNDACIÓN TAMBIÉN (web en línea) http://www.tambien.org/index.php?id=5 (consultado 5-12-2014). 